\title{
ACTIVIDAD HIDROGEOLOGICA DEL SUR DEL PERU, UNA EVALUACION SITUACIONAL DE LAS CUENCAS DE SAMA Y LOCUMBA
}

\author{
HYDROGEOLOGIC ACTIVITY OF SOUTHERN PERU, A \\ SITUATIONAL ASSESSMENT OF SAMA AND \\ LOCUMBA BASINS
}

\begin{abstract}
Alberto Franco $^{1}$, Silvia Ponce ${ }^{2}$, Juan Rodríguez ${ }^{3}$
RESUMEN

Es sabido que Sudamérica presenta una intensa actividad geotermal producto del movimiento de placas en las costas del Océano Pacífico, el geotermalismo en el Perú se da especialmente en la zona sur sobre todo en las Regiones de Arequipa, Moquegua y Tacna, esta última es una de las principales, donde se concentra el 29 por ciento del total nacional. Su influencia puede ser positiva, como podría ser el posible uso como fuente de energía, pero también negativa, por la gran carga de contaminantes que estas realizan sobre las cuencas subyacentes, como son las cuencas de Sama y Locumba, que son motivos de este reporte. Por otro lado, la misma naturaleza a través de mecanismos como la dilución con fuentes de agua de buena calidad, o la floculación con hierro de origen natural, contribuye de modo importante al control de algunos metales contaminantes como el arsénico, que por ejemplo en el caso de la cuenca de sama, mejora su calidad en casi en un cincuenta por ciento. En este artículo, comparamos esta situación y posibles implicancias de su tratamiento para el desarrollo de la región.

Palabras clave.- Hidrogeoquímica, Arsénico, Agua, Cuencas.

ABSTRACT

It is known that South America has an intense geothermal activity product of plate movement on the shores of the Pacific Ocean, the geothermal activity in Peru is especially intense in the southern regions especially in Arequipa, Moquegua and Tacna, the latter is one of the main, which accounts for 29 percent of the national activity. His influence can be positive, as might be the possible use as an energy source, but also negative for the high load of pollutants made on these underlying basins, such as basins and Locumba Sama, which are grounds for this report. Furthermore, the very nature through mechanisms such as dilution with water sources good quality iron flocculation or natural origin, contributes significantly to the control of some contaminating metals such as arsenic, for example in the if Sama basin, improves quality by almost fifty percent. In this article, we compare this situation and its possible implications of treatment in the development of the affected zones.
\end{abstract}

Keywords.- Hydrogeochemical, Arsenic, Water basins.

${ }^{1}$ Ing. Químico, investigador del Cite Agroindustrial, Tacna, Perú, ${ }^{2}$ Dra. Investigadora de la Universidad Nacional de Ingeniería y Profesora investigadora de la Universidad de Lima, Perú, ${ }^{3}$ Dr. Profesor Principal de la Universidad Nacional de Ingeniería, Lima-Perú. 


\section{INTRODUCCION}

La zona sur del Perú es caracterizada por una actividad volcánica importante, propia del circulo de fuego del pacifico. Además esta zona es la cabecera del desierto de Atacama, lo cual la caracteriza como una zona de estrés hídrico. Específicamente, el departamento de Tacna, que colinda con Chile, tiene una provisión de agua insatisfecha y agricultura limitada por la sobreexplotación de sus aguas, principalmente subterráneas. Adicionalmente, la presencia de Arsénico, que de acuerdo a las directivas de la Organización mundial de la Salud no debe sobrepasar concentraciones mayores a $10 \mathrm{ug} / \mathrm{L}$ en aguas de consumo humano (WHO, 2006). En el Perú, desde el 2010 se está siguiendo esta directiva con la excepción de sectores abastecidos con agua de origen geotermal, además se estableció un límite de 50 ug/L para agua de riego. La razón de la existencia de los límites máximos permisibles impuestos es su toxicidad establecida en diferentes reportes científicos (Hopenhayn-Rich et. al., 1996; NG et al. 2003). La presencia del arsénico se ha establecido en diferentes países del mundo y en la región se sabe de su existencia en México, Brasil, Bolivia, Chile, Argentina, entre otros (Bundschuh, J. et al. 2008, 2009, Castro, 2006). En el Perú este problema se conoce desde 1984 (Sancha and Castro, 2001, Castro 2006), sin embargo, hasta donde nosotros sabemos, no existe en la literatura especializada un reporte sobre esta problemática en las Cuencas de Sama y Locumba. En ese sentido, en este trabajo se realiza un primer acercamiento a la problemática de la contaminación hidrogeológica de las cuencas de Sama y Locumba en la Región Tacna, así como una comparación de la situación y características en cada una.

\section{DESCRIPCION GEOGRAFICA DEL AREA DE ESTUDIO}

La Región Tacna se ubica en la parte sur occidental de América del Sur, en el extremo sur del Perú, a 1,348 Km. de Lima; en la cabecera del desierto de Atacama, el segundo desierto más grande del mundo. La extensión territorial de la Región Tacna es de 16,075.89 $\mathrm{km}^{2}$. Tacna se ubica entre las coordenadas $16^{\circ} 58^{\prime}$ y $18^{\circ} 20^{\prime}$ de latitud sur y $69^{\circ} 28^{\prime}$ y $71^{\circ} 02^{\prime}$ de longitud oeste. Limita por el norte con el departamento de Moquegua, por el sur con la República de Chile, por el este con el departamento de Puno, y por el oeste con el Océano Pacífico. En la Fig. 1a, se muestra el mapa satelital del departamento de Tacna del 2001, en el, se muestran las tres cuencas que existen en el departamento: Sama, Locumba y Caplina, la cuenca del Caplina es la más grande y en ella se concentra el 90 por ciento de la población y mayor riqueza agrícola del departamento, La cuenca de Sama y finalmente la cuenca Locumba.

La región Tacna políticamente está constituida por 04 provincias: Tacna, Tarata, Candarave y Jorge Basadre y por 27 distritos. Según el Censo de Población y Vivienda 2007, el número de habitantes es de 288781 , representando el 1 por ciento del total nacional. La población urbana representa el 91 por ciento del total. Sin embargo, como se puede observar en la Tabla 1, la superficie de las tres provincias restantes, en donde vive el 9\% de la población, constituye el $50.25 \%$ de la superficie total. En ese sentido y ante el deterioro de las fuentes subterráneas debido principalmente al sobre consumo agrícola, es importante tener en cuenta la situación de las dos cuencas en cuestión, la cuenca de Sama y la de Locumba.

Tabla $N^{o} 1$ Provincias, extensión y población de Tacna de acuerdo al último Censo Nacional de Población y Vivienda del 2007.

\begin{tabular}{lll}
\hline Provincia & Superficie $\left(\mathrm{km}^{2}\right)$ & Población \\
\hline Tacna & 8066 & 262731 \\
Candarave & 2261 & 8373 \\
Jorge Basadre & 2959 & 9872 \\
Tarata & 2859 & 7805 \\
Total & 16076 & 288781 \\
\hline
\end{tabular}




\section{DESCRIPCION GEOLÓGICA}

En la Fig. 1b se presenta la estructura geológica del departamento de Tacna. La cual se ha hecho coincidir con la del mapa físico de la Fig. 1a. Claramente se observa que el proceso tectónico y volcánico ha dado lugar a una configuración morfológica de varios macroambientes: el fondo marítimo abisal, fosa oceánica, talud, plataforma continental, y cordillera costanera conformada por afloramientos de rocas intrusivas que se elevan hasta los $1.400 \mathrm{msnm}$, y que por fallas va descendiendo hasta perderse en el subsuelo; la repisa continental, valles costeros y la Cordillera Occidental de los Andes. La cual presenta una cantidad importante de volcanes pliocuaternarios ( $-\phi)$, volcanismo continental ( Plutonitas molasas $\mathrm{y}$ volcánicas $(\bullet)$, Fallas inferiores (П), Depósito clástico, volcano clástico, triásico, jurásico, sedimentos continentales volcánicos piocaternarios ( — En la región se nota un claro alineamiento estructural de gran extensión de dirección Norte 45 Oeste. A lo largo de su recorrido fractura las rocas volcánicas del Grupo Toquepala y también a los cuerpos intrusivos; en algunos tramos y uxtapone ambos tipos de rocas. Al parecer, esta falla se encuentra activa pues se tienen reportes de sismicidad con focos de diferentes profundidades a lo largo del alineamiento, y que junto con las evidencias de campo indican que el sistema de fallas Incapuquio conforman el elemento estructural de mayor importancia de la región, pues sería de gran escala en profundidad al punto de atravesar toda la Litosfera, y que ha estado activo desde por lo menos el inicio del período Jurásico (Sempere et al., 2002, 2004). Este rasgo estructural tiene una gran importancia geológica para la comprensión de la geodinámica interna del planeta en la región (Fornari et. al., 2002), que ha producido la formación de la cadena montañosa de los Andes, pero es a su vez es un importante foco de peligros geológicos por la sismicidad, fracturación, y alteración que produce en las rocas asociadas a su actividad, lo cual pone en riesgo permanente los poblados asentados en su área de influencia. Esta situación es reafirmada con la presencia de un gran número de fuentes termales, las cuales son descritas en la Fig. 1. Estas son 169 (Anuario de estadísticas, 2010) de las 589 que existen en todo el país. Esta actividad es sumamente importante y puede ser, eventualmente, utilizada como una fuente de energía geotérmica con posibilidades de desarrollar estas zonas, sin embargo se puede observar, que parte de estas fuentes termales participan directamente de la contaminación de las cuencas de Sama y Locumba. Se hizo muestreos de campo entre enero y marzo del 2008, en los puntos establecidos en el mapa de la Figura 2. Las letras desde A hasta I corresponden a los puntos de la cuenca de Sama y de J hasta Q, la correspondiente a la cuenca de Locumba. Los detalles de las cuencas se muestran en la Tabla 2. La cuenca de Sama es formada por la confluencia de los ríos Estique (A) y Tarucachi (B), que forman el río Aruma (D). Siguiendo el curso del agua y luego que se une el tributario Pistala (C), se forma el rio Tala (E) el cual de su confluencia con el Salado se forma el río Sama (G, H, I). En cuanto a la cuenca de Locumba, esta comienza cuando el otro de los ramales del río Salado (J) se junta con el Callazas y desesembocan en la laguna de Aricota. En esta laguna se forman el rio Curibaya (L, M), el cual al reunirse finalmente con el río Ilabaya $(\mathrm{N})$, se forma el río Locumba $(\mathrm{O}, \mathrm{P}, \mathrm{Q})$. 
(a)

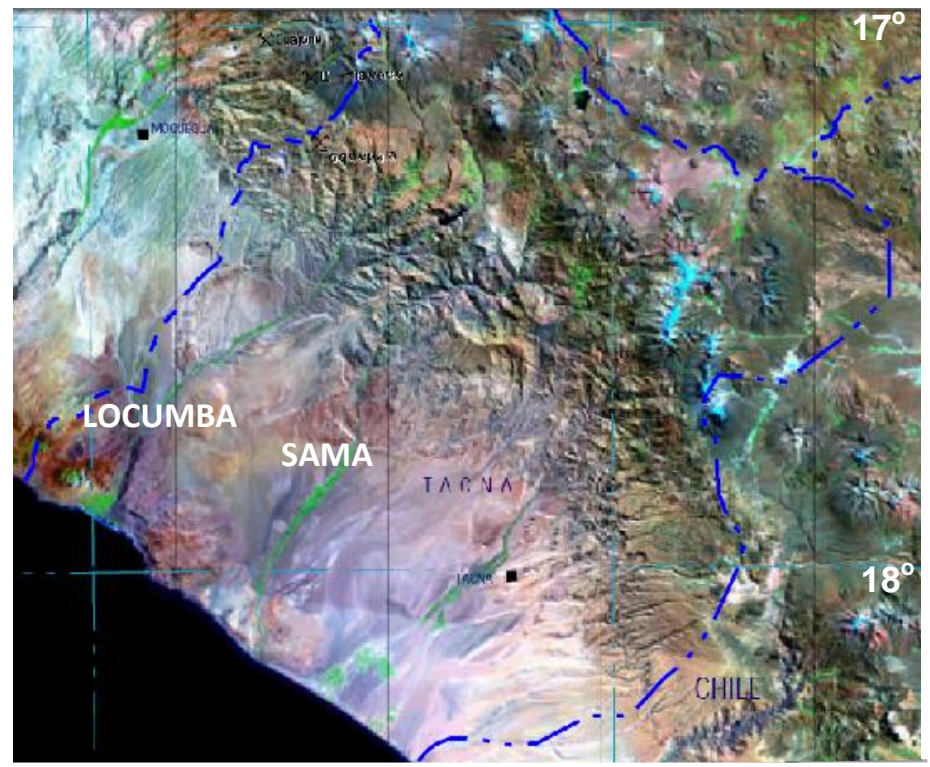

(b)

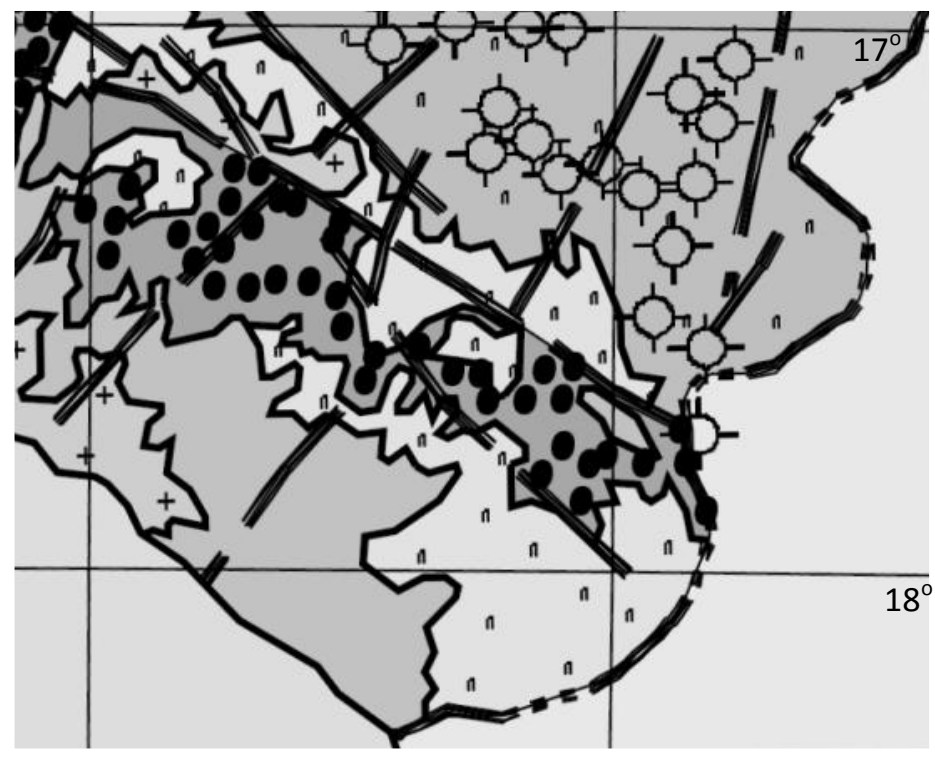

Fig. 1 a) Mapa Físico de Tacna, adaptado del (Atlas M.E.M, 2000), (b) Mapa tectónico de Tacna, adaptado de (Atlas M.E.M, 2000). 


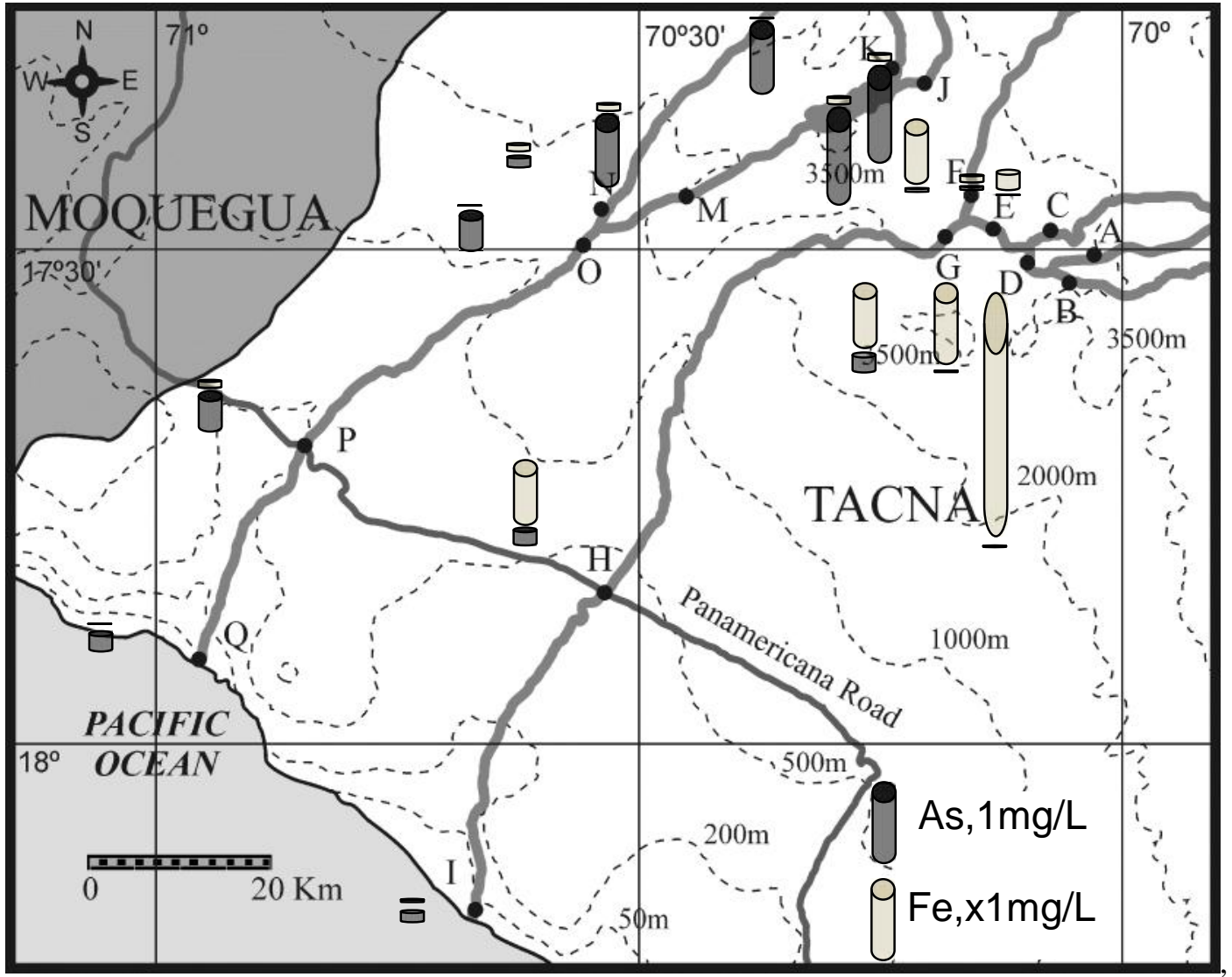

Fig. 2. Mapa de Tacna, mostrando las zonas de muestreo, A-I corresponden a la cuenca de Sama y J-Q a la cuenca de Locumba. Las barras muestran la concentración promedio de Hierro y Arsénico.

\section{MATERIALES Y METODOS}

La toma de muestras realizó bajo la modalidad de la Norma Técnica Peruana N’214-005.

El frasco es sumergido en contracorriente y para los estudios en laboratorio, se aplica una dosis de una gota de Acido Nítrico concentrado por cada litro de muestra para el análisis de metales pesados.

El análisis fisicoquímico se realizó lo antes posible después de recolectada la muestra, los parámetros analizados son los siguientes: $\mathrm{pH}$, Potenciometrico, Conductividad, Conductimetrico, Turbidez, Nefelométrico, Alcalinidad, mediante titulación con ácido Sulfúrico, Bicarbonatos mediantes cálculo, Cloruros utilizando Titulación Nitrato de Plata, Sulfatos mediante análisis espectrofotómetrico $450 \mathrm{~nm}$, Dureza Total y Calcio, mediante Titulación con EDTA, Magnesio se obtuvo por diferencia, Sodio, por absorción atómica.

Todos los químicos utilizados fueron de grado analítico.

Técnicas diversas fueron utilizadas para determinar la concentración de arsénico, se utilizó la voltametría de decapado anódico, con un sistema de microelectrodos de oro de Trace Detect, modelo Nanoband Explorer II, un equipo de absorción atómica con horno de grafito, Shimadzu AAS AA- 6501.

El estudio colorimétrico fue realizado con un espectrofotómetro Thermo Scientific, modelo evolution 300 . 
El boro fue cuantificado utilizando el método colorímetrico del acido carmínico y el Fe (II) fue analizado con 1,10 fenantrolina y la alcalinidad fue medida utilizando el método descrito en (Standard methods, 1985).

\section{RESULTADOS Y DISCUSION}

Los resultados de las muestras colectadas en los puntos especificados en la Tabla 2, son presentados en la Tabla 3. Un promedio de los datos colocados para el Arsénico y para el Hierro en la Figura 3. Se puede observar en este caso que en general en la cuenca de Sama hay una mayor concentración de hierro que en la cuenca de Locumba.

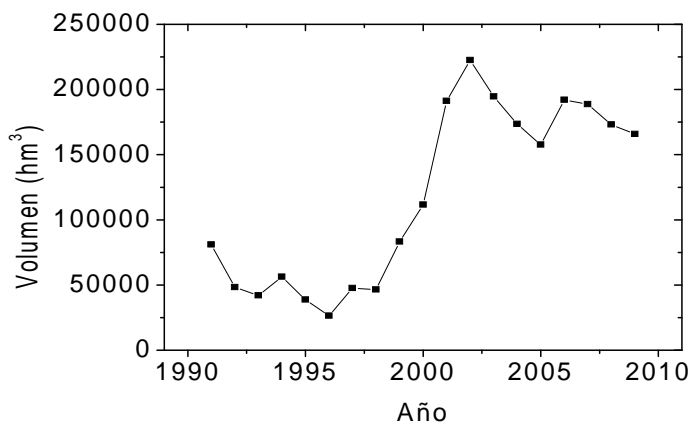

Fig. 3 Promedios anuales de los volúmenes de agua almacenados en la laguna de Aricota en los años mostrados (Compendio estadístico de Tacna, 2009). 
Tabla $N^{o} 2$ Nombre y posición geográfica de los puntos de muestreo en las cuencas de Sama y Locumba.

\begin{tabular}{|c|c|c|c|c|c|}
\hline \multicolumn{3}{|c|}{ Cuenca de Sama } & \multicolumn{3}{|c|}{ Cuenca de Locumba } \\
\hline $\begin{array}{l}\text { Nombr } \\
\text { e }\end{array}$ & $\begin{array}{l}\text { Nombre } \\
\text { del río }\end{array}$ & Posición geográfica & $\begin{array}{l}\text { Nom } \\
\text { bre }\end{array}$ & $\begin{array}{l}\text { Nombre } \\
\text { del río }\end{array}$ & Posición geográfica \\
\hline A & Estique & $\begin{array}{l}17^{\circ} 31^{\prime} 42.00^{\prime \prime S} \\
70^{\circ} 1 ' 54.00^{\prime \prime} \mathrm{W}\end{array}$ & $\mathrm{J}$ & Salado & $\begin{array}{l}17^{\circ} 19^{\prime} 53.58 " \mathrm{~S} \\
70^{\circ} 13^{\prime} 27.00^{\prime \prime} \mathrm{W}\end{array}$ \\
\hline B & Tarucachi & $\begin{array}{l}17^{\circ} 31^{\prime} 18.12^{\prime \prime S} \\
70^{\circ} 2^{\prime} 1.26^{\prime \prime} \mathrm{W}\end{array}$ & $\mathrm{K}$ & Callazas & $17^{\circ} 19^{\prime} 45.00^{\prime \prime S} 70^{\circ} 14^{\prime} 7.98^{\prime \prime W}$ \\
\hline $\mathrm{C}$ & Pistala & $\begin{array}{l}17^{\circ} 29^{\prime} 46.80^{\prime \prime S} \\
70^{\circ} 5^{\prime} 43.02^{\prime \prime} \mathrm{W}\end{array}$ & M & Curibaya & $\begin{array}{l}17^{\circ} 26^{\prime} 17.04^{\prime \prime S} \\
70^{\circ} 25^{\prime} 47.58^{\prime \prime} \mathrm{W}\end{array}$ \\
\hline $\mathrm{D}$ & Aruma & $\begin{array}{l}17^{\circ} 29^{\prime} 57.00 " \mathrm{~S} \\
70^{\circ} 5^{\prime} 39.00^{\prime \prime} \mathrm{W}\end{array}$ & $\mathrm{N}$ & Ilabaya & $17^{\circ} 28^{\prime} 9.00^{\prime \prime S} 70^{\circ} 32^{\prime} 31.80^{\prime \prime} \mathrm{W}$ \\
\hline $\mathrm{E}$ & Tala & $\begin{array}{l}17^{\circ} 28^{\prime} 15.00 " \mathrm{~S} \\
70^{\circ} 9^{\prime} 30.00^{\prime \prime} \mathrm{W}\end{array}$ & $\mathrm{O}$ & Locumba & $\begin{array}{l}17^{\circ} 28^{\prime} 34.62^{\prime \prime S} \\
70^{\circ} 32^{\prime} 33.12^{\prime \prime} \mathrm{W}\end{array}$ \\
\hline $\mathrm{F}$ & Salado & $\begin{array}{l}17^{\circ} 28^{\prime} 13.02^{\prime \prime S} \\
70^{\circ} 10^{\prime} 10.98^{\prime \prime} \mathrm{W}\end{array}$ & $\mathrm{P}$ & Locumba & $17^{\circ} 41^{\prime} 6.72^{\prime \prime S} 70^{\circ} 50^{\prime} 33.90^{\prime \prime} \mathrm{W}$ \\
\hline $\mathrm{G}$ & Sama & $\begin{array}{l}17^{\circ} 28^{\prime} 35.40^{\prime \prime} \mathrm{S} \\
70^{\circ} 10^{\prime} 18.18^{\prime \prime} \mathrm{W}\end{array}$ & Q & Locumba & $\begin{array}{l}17^{\circ} 54^{\prime} 25.68^{\prime \prime S} \\
70^{\circ} 57^{\prime} 28.98^{\prime \prime} \mathrm{W}\end{array}$ \\
\hline $\mathrm{H}$ & Sama & $\begin{array}{l}17^{\circ} 50 ' 31.02^{\prime \prime} \mathrm{S} \\
70^{\circ} 31^{\prime} 12.00^{\prime \prime} \mathrm{W}\end{array}$ & & & \\
\hline I & Sama & $\begin{array}{ll}18^{\circ} & 9 ' 33.00^{\prime \prime S} \\
70^{\circ} 40^{\prime} 10.98^{\prime \prime} \mathrm{W} & \end{array}$ & & & \\
\hline
\end{tabular}


Tabla $N^{o} 3$ Parámetros fisicoquímicos y elementales de cada uno de los puntos de muestreo, realizados en las fechas señaladas.

\begin{tabular}{|c|c|c|c|c|c|c|c|c|c|c|c|c|c|c|c|c|c|}
\hline & & A & B & $\mathrm{C}$ & $\mathrm{D}$ & $\mathrm{E}$ & $\mathrm{F}$ & G & $\mathrm{H}$ & I & $\mathrm{J}$ & $\mathrm{K}$ & $\mathrm{M}$ & $\mathrm{N}$ & $\mathrm{O}$ & $\mathrm{P}$ & Q \\
\hline $\mathrm{pH}$ & $\begin{array}{l}\text { Unida } \\
\mathrm{d}\end{array}$ & 6 & 2.5 & 8.2 & 3.05 & 6.37 & 8.37 & 7.98 & 7.46 & & 8.55 & 9.48 & 8.32 & 8.15 & 8.16 & 8.03 & \\
\hline \multirow[t]{2}{*}{ Conduc } & $\mathrm{Scm}$ & 138 & 116 & 769 & 155 & 109 & 256 & 163 & 263 & & 258 & 120 & 198 & 203 & 162 & 2580 & \\
\hline & 1 & 3 & 0 & & 6 & 8 & 0 & 5 & 0 & & 0 & 8 & 9 & 0 & 0 & & \\
\hline Solid. & $\mathrm{mgL}^{-1}$ & 940 & 845 & 520 & 105 & 738 & 193 & 110 & 175 & & 172 & 803 & 133 & 135 & 108 & 1731 & \\
\hline Tot. & & & & & 0 & & 1 & 4 & 8 & & 4 & & 2 & 8 & 3 & & \\
\hline Bicarbo & $\mathrm{mgL}^{-1}$ & 16.1 & 000 & 64.6 & 000 & 40.4 & 86.9 & 34.0 & 99.0 & & 52.5 & 22.0 & 85.0 & 94.9 & & 119. & \\
\hline nato & $\mathrm{HCO}_{3}$ & 6 & 0.00 & 0 & 0.00 & 0 & 0 & 0 & 0 & & 0 & 0 & 0 & 0 & & 18 & \\
\hline $\begin{array}{l}\text { carbonat } \\
\text { o }\end{array}$ & $\begin{array}{l}\mathrm{mgL}^{-1} \\
\mathrm{CO}_{3}\end{array}$ & 0 & 0 & 0 & 0 & 0 & 0 & 0 & 0 & & 6 & 26 & 4 & 0 & 0 & 0 & \\
\hline $\mathrm{Na}$ & $\mathrm{mgL}^{-1}$ & 31.1 & 17.4 & 43.7 & 42 & 50.3 & 375 & 137 & 254 & & 363 & 154 & 240 & 255 & 158 & 306 & \\
\hline $\mathrm{Fe}, \mathrm{AA}$ & $\mathrm{mgL}^{-1}$ & 0.92 & 0 & 0.24 & 4.01 & 2.53 & 0.25 & 1.86 & 1.78 & & 0.61 & 0.21 & 0.17 & 0.22 & 0.16 & 0.21 & \\
\hline $\begin{array}{l}\mathrm{Fe}, \\
\text { Color }\end{array}$ & $\mathrm{mgL}^{-1}$ & 1.18 & 9.33 & 0.51 & 4.63 & 3.16 & 0.49 & 2.78 & 3.26 & & 0.92 & 0.11 & 0.12 & 0.66 & 0 & 0.25 & \\
\hline Boron & $\mathrm{mgL}^{-1}$ & & & & & & & & & & & & & & & & \\
\hline As, & $\mathrm{mgL}^{-1}$ & 0.01 & 0.01 & 0.08 & 0.00 & 0.03 & 1.28 & 0.40 & 0.17 & & 1.42 & 0.69 & 0.85 & 0.82 & 0.08 & 0.73 & \\
\hline Color & & 1 & 2 & 6 & 9 & 7 & 3 & 0 & 5 & & 5 & 0 & 0 & 6 & 0 & 7 & \\
\hline As, & $\mathrm{mgL}^{-1}$ & 0.01 & 0.01 & 0.12 & 0.01 & 0.07 & 1.55 & 0.51 & 0.20 & & 1.18 & 0.54 & 0.64 & 0.63 & 0.06 & 0.59 & \\
\hline Elect. & & 0 & 8 & 9 & 0 & 5 & 5 & 0 & 6 & & 2 & 3 & 2 & 5 & 7 & 4 & \\
\hline As, & $\mathrm{mgL}^{-1}$ & 0.01 & 0.01 & 0.08 & 0.00 & 0.04 & 1.04 & 0.37 & 0.18 & & 1.22 & 0.81 & 0.82 & 0.85 & 0.08 & 0.67 & \\
\hline Elect. & & 2 & 3 & 7 & 8 & 2 & 3 & 5 & 0 & & 6 & 3 & 2 & 7 & 2 & 3 & \\
\hline AsIII, & $\mathrm{mgL}^{-1}$ & 0.00 & 0.00 & 0.01 & 0.00 & 0.00 & 0.11 & 0.02 & 0.04 & & 0.14 & 0.16 & 0.16 & 0.04 & 0.05 & 0.04 & \\
\hline Elect. & & 3 & 3 & 7 & 6 & 7 & 8 & 9 & 1 & & 9 & 1 & 8 & 1 & 2 & 2 & \\
\hline AsV & $\mathrm{mgL}^{-1}$ & 0.00 & 0.01 & 0.07 & 0.00 & 0.03 & 0.92 & 0.34 & 0.13 & & 1.07 & 0.65 & 0.65 & 0.81 & 0.03 & 0.63 & \\
\hline Elect. & & 9 & 0 & 0 & 2 & 5 & 5 & 6 & 9 & & 7 & 2 & 4 & 6 & 0 & 1 & \\
\hline $\begin{array}{l}08 / 03 / 2 \\
008\end{array}$ & Fecha & & & & & & & & & & & & & & & & \\
\hline
\end{tabular}




\begin{tabular}{|c|c|c|c|c|c|c|c|c|c|c|c|c|c|c|c|c|c|}
\hline & & A & B & $\mathrm{C}$ & $\mathrm{D}$ & $\mathrm{E}$ & $\mathrm{F}$ & G & $\mathrm{H}$ & I & $\mathrm{J}$ & K & M & $\mathrm{N}$ & $\mathrm{O}$ & $\mathrm{P}$ & Q \\
\hline $\mathrm{pH}$ & Unidad & 5.52 & 4.35 & 7.68 & 5.42 & 6.2 & 7.05 & 6.9 & 7.65 & 7.88 & 7.3 & 7.2 & & 7.08 & 7.1 & 7.2 & 7.14 \\
\hline Conduct. & $\mathrm{Scm}^{-1}$ & 532 & 512 & 312 & 522 & 444 & 1680 & 712 & 1102 & 2170 & 1100 & 1220 & & 2224 & 825 & 1380 & 1410 \\
\hline Solid. Tot. & $\mathrm{mgL}^{-1}$ & 263 & 253 & 154 & 258 & 220 & 832 & 352 & 551 & 1080 & 545 & 604 & & 1101 & 408 & 683 & 698 \\
\hline Bicarbonato & $\begin{array}{l}\mathrm{mgL}^{-1} \\
\mathrm{HCO}_{3}\end{array}$ & 12 & 0 & 49 & 6 & 12 & 146 & 37 & 73 & 98 & 73 & 268 & & 207 & 61 & 183 & 275 \\
\hline Cloruros & $\mathrm{mgl}^{-1} \mathrm{Cl}^{-}$ & 38 & 22 & 32 & 27 & 27 & 450 & 105 & 150 & 480 & 245 & 202 & & 380 & 110 & 340 & 421 \\
\hline Sulfatos & $\mathrm{mgl}^{-1} \mathrm{SO} 4$ & 200 & 230 & 80 & 220 & 170 & 125 & 160 & 269 & 316 & 125 & 215 & & 310 & 220 & 290 & 392 \\
\hline Dureza Total. & $\begin{array}{l}\mathrm{mgl}^{-1} \\
\mathrm{CaCO}_{3}\end{array}$ & 250 & 200 & 110 & 230 & 180 & 300 & 220 & 340 & 640 & 170 & 250 & & 450 & 280 & 440 & 550 \\
\hline $\mathrm{Ca}$ & $\mathrm{mgl}^{-1}$ & 81 & 61 & 36 & 73 & 57 & 89 & 61 & 105 & 194 & 40 & 69 & & 101 & 81 & 97 & 141 \\
\hline $\mathrm{Mg}$ & $\mathrm{mgl}^{-1}$ & 12 & 12 & 5 & 12 & 10 & 19 & 17 & 19 & 38 & 17 & 19 & & 48 & 19 & 48 & 48 \\
\hline $\mathrm{Na}$ & $\mathrm{mgl}^{-1}$ & 6 & 20 & 16 & 11 & 15 & 192 & 48 & 75 & 158 & 150 & 142 & & 261 & 52 & 99 & 61 \\
\hline B & $\mathrm{mgl}^{-1}$ & $<0.1$ & 0.1 & 0.2 & 0.1 & 0.2 & 6.1 & 0.8 & 1.7 & 5.6 & 4.8 & 4.8 & & 9.8 & 2.2 & 5.8 & 5.9 \\
\hline $\mathrm{Fe}$, Color & $\mathrm{mgl}^{-1}$ & 0.65 & 12.40 & 0.21 & 5.42 & 0.52 & 0.03 & 0.32 & 0.25 & 0.15 & & & & & & & \\
\hline $\begin{array}{l}\text { As, Color } \\
18 / 01 / 2008\end{array}$ & $\begin{array}{l}\mathrm{mgl}^{-1} \\
\text { Fecha }\end{array}$ & 0.002 & 0.008 & 0.052 & 0.011 & 0.046 & 0.821 & 0.272 & 0.145 & 0.229 & 0.902 & 0.902 & & 0.918 & 0.092 & 0.445 & 0.412 \\
\hline
\end{tabular}




\section{RIO SAMA}

La cuenca Sama, en lo que a $\mathrm{pH}$ se refiere, se encuentra dividido en dos grandes sectores, principalmente el conformado por el sector del lado de las microcuencas de Estique y Tarucachi, con pHs muy bajos, llegando en algunas oportunidades a 1.5 unidades, debido principalmente a la saturación de $\mathrm{CO}_{2}$ expresada como acidez, con el recorrer de las aguas a través de la cuenca el contenido de carbonatos en el suelo hace que el $\mathrm{pH}$ se vaya incrementando progresivamente hasta su mezcla con el río Pistala con $\mathrm{pH}$ por encima de 7. Seguidamente con la unión del río Pistala y Aruma se forma el río Tala y con la unión del río Salado se forma el río Sama en el puente de Talapalca, por lo tanto el $\mathrm{pH}$ del río Sama mucho depende del caudal de ambos ríos. El contenido de hierro en el agua, por otro lado, está determinado por la concentración de este elemento en los ríos Estique y Tarucachi, diluyéndose con el ingreso de todos los demás afluentes del río Sama. El rápido decrecimiento de los contenidos de hierro, se pueden deber a procesos de dilución y a la insolubilidad del mismo, lo cual produce una rápida precipitación de las sales de hierro, conforme el $\mathrm{pH}$ se incrementa a lo largo del recorrido del río. En la formación de los manantiales por el sector Tarucachi y Estique las concentraciones de hierro llegan a valores por encima de $50 \mathrm{mg} / \mathrm{l} \mathrm{Fe}$, sin embargo este elemento va diluyendo su concentración hasta llegar a valores de $0.12 \mathrm{mg} / \mathrm{l} \mathrm{Fe}$.

El contenido de Arsénico en el río Sama está determinado por la concentración de este elemento en el río Salado, el río Tala contiene valores muy bajos de Arsénico provenientes de los ríos Pistala y Aruma. El río Salado al mezclarse con las aguas del río Tala diluye su concentración a valores de 0.25 a $0.30 \mathrm{mg} / \mathrm{l}$ As, y que van precipitando progresivamente hasta llegar a la Boca del Río Sama. Aun así los contenidos de Arsénico a lo largo del río Sama son elevados, y sobrepasan la normatividad para aguas de consumo humano.

Por otro lado, el contenido de Boro está determinado por la concertación de este elemento presente en mayor porcentaje del río Salado. El río Salado en periodos de lluvia presenta una concentración de $6 \mathrm{mg} / \mathrm{l}$ B y se diluye con las aguas del río Tala que presenta concentraciones de $0.2 \mathrm{mg} / \mathrm{l} \mathrm{B}$. A medida que discurren sus aguas

TECNIA 22 (1) 2012 desde el puente Tomasiri el contenido de Boro va creciendo hasta llegar al mar, esto ocurre por el lavado de sales de los suelos por donde pasa el río, que contienen depósitos de sales llamados estratos salinos con altos contenidos de Boro, en forma de tetraboratos de sodio.

\section{RIO LOCUMBA}

En la Tabla 3 se puede observar que los afluentes de la laguna de Aricota son básicos debido a que el recorrido de estos es relativamente largo, el $\mathrm{pH}$ de los manantiales contaminados en estos sectores sobre todo en el sector Calientes Yucamani son ligeramente ácidos, a medida que el río discurre por las quebradas la alcalinidad se va incrementando por la presencia de carbonatos en el suelo y por la liberación del $\mathrm{CO}_{2}$ contenido en el agua presurizada de los manantiales. El proceso de transformación de los bicarbonatos en el agua es adscrito a bacterias que en su proceso de metabolismo convierten este compuesto en carbonatos incrementando el $\mathrm{pH}$. Durante el proceso de sedimentación natural que se da en la laguna Aricota, el $\mathrm{pH}$ se incrementa hasta valores de 8.2 y valores mayores dependiendo del periodo del año, por otro lado cuando entra el río Ilabaya con aguas de lluvia el $\mathrm{pH}$ se reduce por dilución luego se va incrementando a lo largo del río por el arrastre de bicarbonatos y carbonatos. El contenido de Hierro en esta cuenca es muy bajo, siendo el río salado el que mayor concentración presenta con $0.6 \mathrm{mgL}^{-1}$. Por otro lado el arsénico, está presente en el río Callazas y en el río Salado, desde la formación del río Callazas existen manantiales hidrotermales que presentan contenidos muy elevados de este elemento, así mismo discurren por las quebradas manantiales de buena calidad y aguas de deshielo que ayudan a la dilución de sales. Todos estos manantiales se reúnen y forman el río Calientes, el cual actualmente está siendo captado casi en su totalidad para fines agrícolas. La laguna de Aricota se observa como un sistema de control de la contaminación, ya que entran en promedio concentraciones de alrededor $1.4 \mathrm{mgL}^{-1}$ del río salado y $0.69 \mathrm{mgL}^{-1}$ del río Callazas y se obtienen a la salida de la laguna, concentraciones de $0.8 \mathrm{mgL}^{-1}$.

Este efecto benéfico es importante mantener, pero esto implica evitar el decrecimiento de los volúmenes de agua o incrementarlos con los de otras fuentes de agua de buena calidad. Es 
necesario resaltar que la laguna de Aricota en 1996 alcanzó su mínimo histórico de $26,415 \mathrm{hm}^{3}$, esta situación se comenzó a revertir y el 2002 alcanzó los $222,485 \mathrm{hm}^{3}$, sin embargo a partir de ese año, como se puede observar en la Fig. 3, el volumen paulatinamente ha comenzado a decrecer, llegando en mayo del 2009 a 165,779 $\mathrm{hm}^{3}$. Esta situación debe revertirse pues la eficiencia de precipitación natural mostrada va a decrecer a medida que decrezca el volumen de agua contenido, el cual está muy lejos del máximo histórico alcanzado en 1976, con más de $800,000 \mathrm{hm}^{3}$.

Una manera de analizar la concentración del arsénico con la estación, es hacer una comparación con las lluvias medidas en la zona, en este caso en la estación de Talacaya en Locumba. En la Figura 4 se presentan resultados de la concentración de arsénico en los puntos M, P y $\mathrm{H}$ (las líneas horizontales llenas y punteadas representan las cotas máximas y mínimas de concentración de arsénico, respectivamente). Se puede observar que en general y durante la época de lluvias hay una reducción sistemática de la concentración de arsénico, mientras que este efecto de dilución desaparece en la época de estío en donde la concentración de arsénico vuelve a incrementarse. Tal efecto se observa en ambas cuencas.

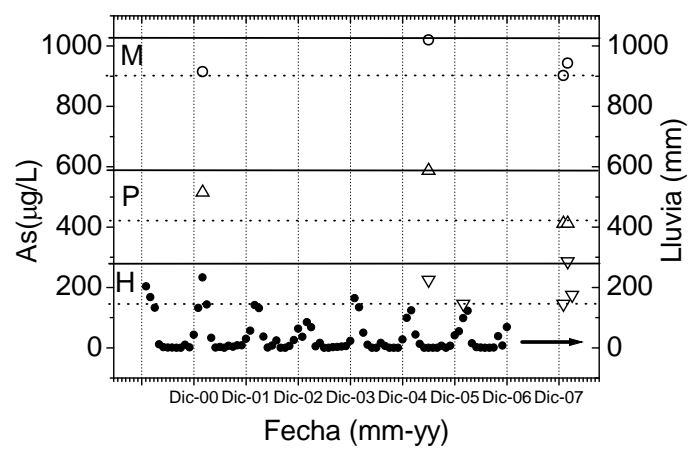

Fig. 4 Concentración de arsénico en los puntos $M, P$ y $H$ y lluvia contabilizada por la estación de Talacaya en los años mostrados. Los datos de arsénico entre el 2001 y el 2006 corresponden al proyecto especial Tacna.

Finalmente, el boro es un elemento presente en todos los procesos hidrotermales, es por ello que su contenido demarca una característica especial en los bordes de los ríos con altos contenidos de estas sales, en los manantiales más contaminados los contenidos llegan a $60-70 \mathrm{mg} / \mathrm{l} \mathrm{B}$, y se diluyen hasta llegar a la laguna de Aricota. En la laguna de Aricota ocurre un proceso de precipitación de sales sin embargo se ha notado que los contenidos de Boro no precipitan tanto como el Arsénico debido a que el Boro es más liviano que el Arsénico y también el Boro tiende a diluirse como acido bórico en el agua haciéndolo muy soluble.

\section{CONCLUSIONES}

Las cuencas de Sama y Locumba presentan concentración elevadas de arsénico y boro, producto de la actividad hidrotermal en la cordillera de los andes. La concentración de los contaminantes es dependiente de la época del año y disminuyendo en épocas de lluvia. La concentración del arsénico en la cuenca de Sama se reduce por efecto de dilución y de la presencia de hierro en alguno de los tributarios. El arsénico identificado es mayormente arsénico de valencia (V), frente al arsénico (III) que está en una proporción un decimo menor.

Los contenidos de boro no decrecen en concentración a lo largo de la cuenca, presentan este comportamiento por su mayor poder de dilución. La Laguna de Aricota es un sistema natural de precipitación en la cuenca de Locumba, que sin embargo debe de ser adecuadamente gestionada para que su volumen se incremente en el tiempo.

Este trabajo en esta primera etapa, pretende explorar nuevas alternativas de control de la contaminación de las cuencas de Sama y Locumba, como es la adecuada gestión del recurso, como una manera de incrementar la actividad agrícola, además de mejorar la calidad de vida de la población.

\section{AGRADECIMIENTOS}

Este trabajo ha sido financiado con el proyecto Fincyt PIBAP 22, 2007. Los autores agradecen al Proyecto Especial Tacna por el apoyo brindado. Uno de los autores J. Rodríguez, agradece al Convenio de Desempeño UTA-MINEDUC por el soporte financiero. 


\section{REFERENCIAS}

1. Instituto Nacional de Estadística e Informática, INEI "Anuario de estadísticas ambientales", Lima, 2010.

2. Ministerio de Energía y Minas "Atlas de minería y energía en el Perú”, 2001.

3. Oficina Departamental de Estadística e Informática "Compendio estadístico departamental de Tacna", Diciembre 2009,Tacna, 29,30.

4. Bundschuh, J., Pérez Carrera, A., Litter, M. I., "Distribución del arsénico en la región Ibérica e Iberoamericana". CYTED, Argentina Available, (Eds.), 2008 in: http://www.cnea.gov.ar/xxi/ambiental/iberoar sen/default.asp (In Spanish).

5. Bundschuh, J., Armienta, M.A., Bhattacharya, P., Matschullat, J., Birkle, P., Mukherjee, A.B. (Eds.), 2009. Natural Arsenic in Groundwater of Latin America Occurrence, Health Impact and Remediation. Balkema Publisher, Lisse, the Netherlands.

6. Castro de Esparza, M. L., 2006. The presence of arsenic in drinking water in Latin America and its effect on public health. In: J. Bundschuh, M.A. Armienta, P. Bhattacharya, J. Matschullat, P. Birkle, y R. Rodríguez (eds): Natural arsenic in groundwaters of Latinoamerica. Proceedings of the International Congress on Natural Arsenic in Groundwaters of Latinamerica, México, pp. 3-6

7. Fornari, M., Mamani, M., Ibarra I., Carlier G., Datación del periodo volcánico "Tacaza" en el Altiplano de Perú y Bolivia. Resumen, XI Congreso Peruano de Geología, Lima, p. 21. 2002.

8. Hopenhayn-Rich, C., Biggs, M. L., Fuchs, A., Bergoglio, R., Tello, E. E., Nicolli, H., Smith, A. H., Bladder cancer mortality associated with arsenic in drinking water in Argentina. Epidemiol. 7, 117-124. 1996.
9. Ng, J.C, Wang, J., Shraim, A, “A global health problem caused by arsenic from natural sources", Chemosphere 52 13531359, 2003.

10. Nordstrom D. K., Worldwide occurrences of arsenic in ground water. Science, 296, 2143-2145, 2002.

11. World Health Organization, "Guidelines for deinking water quality", ISBN 924 1546962006. http://www.who.int/water_sanitation_health/ dwq/gdwq3rev/en/

12. Sancha, A. M., Castro, M. L., Arsenic in Latin America: occurrence, exposure, health effects, and remediation. In: Chappell, W.R., Abernathy, C.O., Calderon, R.L. (Eds.), Arsenic Exposure and Health Effects. Elsevier, pp. 87-96, 2001.

13. Sempere, T., Carlier, G., Soler, P., Fornari, M., Carlotto, V., Jacay, J., Arispe, O., Néraudeau, D., Cárdenas, J., Rosas, S., Jiménez, N., "Late PermianMiddle Jurassic lithospheric thinning in Peru and Bolivia, and its bearing on Andean-age tectonics", Tectonophysics 345, 153-181, 2002.

14. Sempere, T, Jacay, J., Carlotto, V., Martinez, W., Bedoya, C., Fornari, M, Roperch, P., Acosta, H., Acosta, J., Cerpa, L., Flores, A., Ibarra, I., Latorre, O., Mamani, M., Meza, P., Odonne F., Oros, Y., Pino. A., Rodriguez R., Sistemas Transcurrentes de escala litosférica en el sur del Perú, Publicación Especial SGP WS, 105-110, 2004. http://horizon.documentation.ird.fr/exldoc/pleins_textes/divers1006/010036851.pdf,

15. Standard Methods for Examination of Water and Wastewater, 16th ed. Washington, DC: APHA, WWA, WPCF, 1985.

Correspondencia: jrodriguez@uni.edu.pe 